\title{
Managing economic and virtual economic capital within financial conglomerates*
}

\author{
Marc J. Goovaerts ${ }^{\dagger \dagger} \bullet$ Eddy van den Borre ${ }^{\dagger \S} \bullet$ Roger J.A. Laeven ${ }^{\ddagger \uparrow}$
}

October 21, 2003

\begin{abstract}
In the present contribution we show how the optimal amount of economic capital can be derived such that it minimizes the economic cost of risk-bearing. The economic cost of risk-bearing takes into account the cost of the economic capital as well as the cost of the residual risk. In addition to the absolute problem of the determination of the amount of economic capital, we also consider the relative problem of how to establish the allocation of economic capital among subsidiaries. However, since subsidiaries are juridical entities they will also solve the problem of economic capital allocation themselves. Clearly, in an equilibrium situation the relative allocation derived by the conglomerate and the absolute allocation derived by the subsidiaries coincide. We show that the diversification benefit which is typically obtained in a conglomerate construction, creates a virtual economic capital for subsidiaries within the conglomerate. We show furthermore that the absolute allocation approach can also be applied to the problem of optimal portfolio selection, extending the well-known Markovitz approach and providing a tool for management by economic capital.
\end{abstract}

Keywords: Risk measurement, Capital allocation, Optimal portfolio selection

JEL-Classification: G22, G31

${ }^{*}$ We are grateful to Jan Dhaene and Rob Kaas for valuable comments. We thank Hans de Cuyper and Johan Daemen for interesting discussions within the Actuarial Contact Program. The views expressed are those of the authors and not necessarily those of Fortis Bank Insurance or Mercer Oliver Wyman. Please address correspondence to Goovaerts: E-mail: Marc.Goovaerts@econ.kuleuven.ac.be

${ }^{\dagger}$ Catholic University of Leuven, Center for Risk and Insurance Studies, Naamsestraat 69, B-3000 Leuven, Belgium.

${ }^{\ddagger}$ University of Amsterdam, Dept. of Quantitative Economics, Roetersstraat 11, 1018 WB Amsterdam, The Netherlands.

${ }^{\S}$ Fortis Bank Insurance, Corporate Actuarial Control, Wolvengracht 48, 1000 Brussel, Belgium.

${ }^{\top}$ Mercer Oliver Wyman, Startbaan 6, 1185 XR Amstelveen, The Netherlands. 


\section{Introduction: the evolution of beliefs}

In many countries an important technique for the financing of life insurances, in particular legal pensions, has been the so-called repartition system, in which the "belief" in the labor force of the next generation "guarantees" the benefits to be paid out. Another "belief" arose when actuaries started to create solvency buffers and to set up reserves, which are financed out of the premiums, in order to be able to pay the benefits in future years, leading to management by economic capital. In that case the "belief" in management activity of the future generations is the "guarantee". A third "belief" was created when investors could convince risk bearers that risk can be hedged within financial markets. Here the "guarantee" is based on the "belief" that in the financial markets one always finds the possibility to place the risk at a predetermined price. These three types of beliefs governing repartition, funding and financial market transfers all contain their own exposure to a default of the approach. For repartition it is clear that longevity is a threat, for funding inflation and other economic factors constitute a threat and in financial markets a breakdown of the system because of e.g. comonotonic effects is a threat, as was indicated recently by Warren Buffett.

Hence, due to the differences in underlying threats, the existence of a perfect hedge between financing structures in financial markets and insurance markets seems to be a fiction. A particular financing structure is based on a belief that can be formulated as a set of axioms, concerning the behavior of rational human beings (see Markovitz $(1952,1959))$. We completely agree with the Markovitz concept and the basic principles that are underlying it: "We might decide that in one context one basic set of principles is appropriate, while in another context a different set of principles should be used. We might find that some patterns of preferences are consistent with a set of preferences while other patterns are not (Markovitz (1959))." Following his flow of ideas, we think that preference axioms constitute flexible definitions to determine or estimate the cost of decisions made in a particular context. This cost depends on an estimate of the risks by means of an axiomatically founded risk measure expressed in financial terms. These types of risk measures have been studied extensively in actuarial science (see e.g. Bühlmann (1970), Gerber (1979) and Goovaerts, De Vylder and Haezendonck (1984)), where they are known as "premium principles", once called "insurance principles" by Markovitz. Variables such as solvency margin, economic capital, capital allocation and asset mix should then be derived in an optimization procedure, e.g. by minimizing economic cost or by maximizing profits, given the preference patterns in the particular context.

Indeed, there exists a fundamental difference between financial markets and insurance markets. In standardized financial markets the law-of-one-price enforces that each risk can be placed in the market at a unique price. This is however certainly not the case in insurance markets, in which generally no unique price exists. The fair value of an insurance portfolio, which is the value of the portfolio induced by market parameters, might be different from the valuation by a particular insurer. Arbitrage opportunities in the illiquid and non-standardized insurance markets are difficult to determine and even more difficult to exploit. Indeed the seller and the buyer may well have a different perception of the price of the portfolio. This is then typically 
due to a different approach to determine the amount of reserves needed. In the case of Value-at-Risk (VaR) e.g., the seller might use a percentile of order $90 \%$ while the buyer uses a percentile of order $95 \%$. One can buy a particular type of car everywhere at almost the same price, but an insurance portfolio is a unique product that can be valued differently according to a management criterion, the degree of risk aversion being the differentiating factor. Different degrees of risk aversion lead to different amounts of economic capital, obtained as a tradeoff between the preferred level of risk exposure and the economic cost of capital. We encounter a similar problem in statistics where there is no unique level to be used in the testing of hypothesis. The reason for this is that hypothesis testing is not a one dimensional problem (with one criterion) but it is a problem where there are two possible errors to be considered: the so-called type I error where one accepts the wrong hypothesis and the type II error where one does not accept the true hypothesis. Indeed, the same type of problem arises in problems of capital allocation and solvency measurement. It is easy to derive a solvency margin that guarantees non-ruin. Clearly, it is sufficient to have an infinite solvency margin. However, the cost of this solvency margin is generally infinite. Hence, the optimal solvency margin is obtained as a compromise between the economic cost of capital on the one hand and the preferred safety level on the other hand.

For the allocation of economic capital one has to consider two different problems. First, one needs to determine how large a solvency margin should be for a given conglomerate. This is the absolute problem of the determination of the economic capital. The second problem concerns how one should allocate a given amount of economic capital (eventually the optimal one in absolute terms) among the different subsidiaries. This is the relative problem of economic capital allocation. Since subsidiaries are legal entities, they will also consider the absolute problem of economic capital allocation themselves. An equilibrium situation exists within the conglomerate in case the relative allocation of economic capital derived by the conglomerate and the absolute allocation derived by the subsidiaries coincide. The widely observed preference for a conglomerate construction can be explained by the existence of a virtual economic capital for subsidiaries within the conglomerate.

In the present contribution we consider the management of economic capital within financial conglomerates. In particular, we address allocation and diversification issues and demonstrate the emergence of virtual economic capital within conglomerates. Furthermore, we discuss the role of economic capital in problems of optimal portfolio selection. We refer to Goovaerts, Dhaene and Kaas (2003) for previous related results. The outline of the paper is as follows: in section 2 we derive solutions to both the absolute and the relative problem of economic capital allocation. In section 3 we compare subsidiaries when incorporated in a conglomerate construction with subsidiaries when considered as stand alone entities and introduce the notion of virtual economic capital. Section 4 demonstrates that the absolute allocation approach can also be applied to the problem of optimal portfolio selection, taking into account the available amount of surplus capital and thus extending the well-known Markovitz approach. 


\section{Capital allocation and solvency measurement}

A risk is represented by a random variable $X$, which is defined on a set of states of nature $\Omega$ and is to be interpreted as the future net loss or deficit of a portfolio or position currently held. For simplicity we henceforth restrict to random variables with absolutely continuous and strictly increasing distribution functions, although the results can be generalized to allow for random variables with discontinuous and non-decreasing distribution functions.

We will first illustrate that from a mathematical point of view the relative problem of optimal allocation of economic capital is equivalent to the relative problem of imposing solvency margins by a regulatory authority. For the former problem, consider a financial conglomerate, in particular think of an insurance company, which consists of $n$ subsidiaries. For the latter problem consider a particular insurance market, consisting of $n$ conglomerates supervised by a regulator. Indeed, letting $X_{j}$ denote the risk of subsidiary $j$, respectively the risk of conglomerate $j, u$ the economic capital, respectively the aggregated solvency capital in the market, the following figures illustrate the same problem:

Figure 1: Capital allocation among subsidiaries

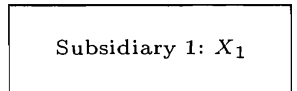

$u_{1}$

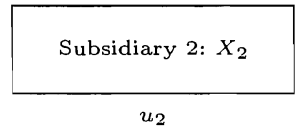

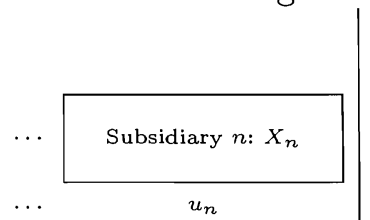

$u_{n}$
Conglomerate: $X_{1}+\ldots+X_{n}$

$u$

The relative capital allocation problem is concerned with the question how the economic capital $u$ is to be distributed among the subsidiaries, attaching $u_{j}$ to subsidiary $j$ in an optimal way. Clearly, the absolute amount of economic capital $u$ strongly depends on the price of economic capital and the risk aversion of the conglomerate.

Figure 2: Solvency measurement by a regulatory authority

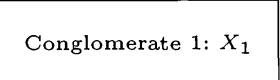

$u_{1}$

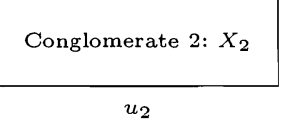

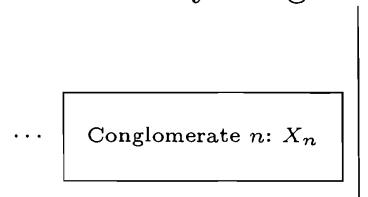

$u_{n}$
Market supervised: $X_{1}+\ldots+X_{n}$

$u$

The relative problem of solvency measurement from a regulatory point of view, is concerned with the question how to attribute to the conglomerates the aggregated solvency capital $u$, which is available in the market, attaching $u_{j}$ to conglomerate $j$ in an optimal way. The value of $u$ in absolute terms highly depends on the risk perception of the conglomerates and the judgement of the regulator.

We remark that both relative problems are important from performance and risk evaluation perspectives. By determining $u_{j}$ for $j=1, \ldots, n$, the conglomerate 
may evaluate the economic cost of risk-bearing and the risk-adjusted return per subsidiary and may allocate costs among subsidiaries correspondingly. The supervisory authority may rank companies on the basis of the ratio of $u_{j}$ and their actually held solvency margin.

There are other problems in insurance which have the same structure. Indeed consider a top-down approach for rating purposes. Suppose one has to fix the total premium income required for a particular insurance portfolio (e.g. determined by market and competition conditions or on statistical grounds). Then the relative problem consists in the distribution of the total premium income over all contracts. Another example is the problem of the determination of optimal reinsurance contracts, where e.g. in proportional reinsurance the relative problem consists in the determination of the different proportionality levels for the different contracts and then match the results with the aggregated required level based on stability criteria (e.g. by using a ruin probability risk measure as in Bühlmann (1970)).

In this section, we will consider both the absolute and the relative problem of economic capital allocation. We demonstrate that there will generally exist optimal values $\left(u^{*}, u_{1}^{* s}, \ldots, u_{n}^{* s}\right)$ which solve the absolute economic capital problem for the conglomerate, respectively for the subsidiaries when considered as stand alone entities. The relative problem consists in the distribution of $u^{*}$ among the subsidiaries allocating $u_{j}^{*}$ to subsidiary $j$, under the constraint that $u^{*}=u_{1}^{*}+\ldots+u_{n}^{*}$. The solutions $\left(u_{1}^{* s}, \ldots, u_{n}^{* s}\right)$ and $\left(u_{1}^{*}, \ldots, u_{n}^{*}\right)$ generally differ.

\subsection{The relative problem of economic capital allocation}

For the relative problem of economic capital allocation, we distinguish two approaches. The first "pragmatic" one consists in defining a risk measure $\rho(X)$, which distributes the capital $u^{*}$ in a proportional way:

$$
\frac{u^{*}}{\rho\left(X_{1}+\ldots+X_{n}\right)}=\frac{u_{1}^{*}}{\rho\left(X_{1}\right)}=\ldots=\frac{u_{n}^{*}}{\rho\left(X_{n}\right)}=\frac{u_{1}^{*}+\ldots+u_{n}^{*}}{\rho\left(X_{1}\right)+\ldots+\rho\left(X_{n}\right)}
$$

One can translate the allocation principle $\rho(\cdot)$ establishing the distribution of economic capital by means of a set of axioms. Indeed, if full allocation of the economic capital is required, i.e. $u_{1}^{*}+\ldots+u_{n}^{*}=u^{*}$, then the axiom of additivity of the risk measure is the axiomatic translation of the above proportional allocation.

The second approach, which is the better one in our opinion, consists in considering the residual risk of each of the subsidiaries. In this approach one has to relate the residual risk of the conglomerate, given by

$$
\max \left(X_{1}+\ldots+X_{n}-u^{*}, 0\right)=\left(X_{1}+\ldots+X_{n}-u^{*}\right)_{+}
$$

to the residual risk of the subsidiaries seen as separated juridical entities, given by

$$
\max \left(X_{1}-u_{1}, 0\right)+\ldots+\max \left(X_{n}-u_{n}, 0\right)=\left(X_{1}-u_{1}\right)_{+}+\ldots+\left(X_{n}-u_{n}\right)_{+}
$$

As long as $u^{*} \geq u_{1}+\ldots+u_{n}$ the diversification effect, being the situation that the conglomerate has a residual risk that is smaller than the one of the subsidiaries, follows because of the following stochastic dominance relation:

$$
\left(X_{1}+\ldots+X_{n}-u^{*}\right)_{+} \leq_{1}\left(X_{1}-u_{1}\right)_{+}+\ldots+\left(X_{n}-u_{n}\right)_{+}, \quad u^{*} \geq u_{1}+\ldots+u_{n}
$$


Hence, any risk measure that preserves stochastic dominance is consistent with the diversification effect. The advantage of considering the residual risk is that we have the opportunity to "optimize" the capital allocation rather than using a proportionality rule which is not based on any economic motivation. When management is facing the problem of allocating the amount $u^{*}$ among the subsidiaries, it is natural to require $u^{*}=u_{1}^{*}+\ldots+u_{n}^{*}$. Consequently the application of the proportional approach would immediately lead to the necessity of having an additive risk measure

$$
\rho\left(X_{1}+\ldots+X_{n}\right)=\rho\left(X_{1}\right)+\ldots+\rho\left(X_{n}\right) .
$$

However, imposing additivity of the risk measure $\rho(\cdot)$ for all forms of dependence structure between the subsidiaries, characterizes an expectation principle under very general conditions (see e.g. Goovaerts, De Vylder and Haezendonck (1984)).

Using the second approach, a "better" distribution of the capital $u$ is possible so as to minimize the residual risk of the subsidiaries. To illustrate the approach, let's take as an example the expectation to measure the residual risk (beware of the distinction between the allocation principle $\rho(\cdot)$, which in fact equals the allocation, and the measure for the risk residual, which is used to establish the allocation). It is obvious that the expectation is a stochastic dominance preserving risk measure and therefore preserves the so-called diversification effects. Then the risk of the subsidiaries as measured by the conglomerate is given by

$$
\mathbb{E}\left[\left(X_{1}-u_{1}\right)_{+}+\ldots+\left(X_{n}-u_{n}\right)_{+}\right]
$$

We then solve the problem

$$
\min _{u_{1}, \ldots, u_{n} \mid \sum_{j=1}^{n} u_{j}=u^{*}} \mathbb{E}\left[\sum_{j=1}^{n}\left(X_{j}-u_{j}\right)_{+}\right]
$$

which minimizes the risk measure (here the expectation) applied to the sum of the risk residuals representing the subsidiaries after the capital allocation has been performed. Solving this problem by means of Lagrange multipliers gives

$$
u_{j}^{*}=F_{X_{j}}^{-1}(1-s), \quad j=1, \ldots, n
$$

where $s$ is determined as $F_{X_{1}^{c}+\ldots+X_{n}^{c}}\left(u^{*}\right)=1-s$, in which $F_{X_{1}^{c}+\ldots+X_{n}^{c}}$ denotes the distribution function of the comonotonic random vector $\left(X_{1}^{c}, \ldots, X_{n}^{c}\right)$ with same marginal distribution functions as $\left(X_{1}, \ldots, X_{n}\right)$. The interested reader is referred to Dhaene et al. (2002a, 2002b) for an elaborate treatment of the concept of comonotonicity. Hence, $u_{j}^{*}=F_{X_{j}}^{-1}\left(F_{X_{1}^{c}+\ldots+X_{n}^{c}}\left(u^{*}\right)\right)$. The expectation of the residual risk of the conglomerate is bounded from above by the expectation of the sum over the residual risks of the subsidiaries:

$$
\mathbb{E}\left[\left(X_{1}+\ldots+X_{n}-u^{*}\right)_{+}\right] \leq \mathbb{E}\left[\sum_{j=1}^{n}\left(X_{j}-F_{X_{j}}^{-1}\left(F_{X_{1}^{c}+\ldots+X_{n}^{c}}\left(u^{*}\right)\right)\right)_{+}\right]
$$


Furthermore, we have that

$$
\begin{array}{r}
\mathbb{E}\left[\left(X_{1}+\ldots+X_{n}-u^{*}\right)_{+}\right] \leq \sum_{j=1}^{n} \mathbb{E}\left[\left(X_{j}-F_{X_{j}}^{-1}\left(F_{X_{1}^{c}+\ldots+X_{n}^{c}}\left(u^{*}\right)\right)\right)_{+}\right]= \\
\mathbb{E}\left[\left(X_{1}^{c}+\ldots+X_{n}^{c}-u^{*}\right)_{+}\right]
\end{array}
$$

Hence, the diversification benefit (DB) of incorporating subsidiaries in a conglomerate is given by

$$
\mathrm{DB}=\mathbb{E}\left[\left(X_{1}^{c}+\ldots+X_{n}^{c}-u^{*}\right)_{+}\right]-\mathbb{E}\left[\left(X_{1}+\ldots+X_{n}-u^{*}\right)_{+}\right]
$$

Note that the diversification benefit stems from the diversification effect on the residual risk level of the conglomerate (see also in this context the report of the Casualty Actuarial Society (1999)). For the expectation risk measure, the diversification benefit is non-negative. Consequently, one might consider a diversification gain to be flowing back to the subsidiaries. A natural choice for the allocation of the diversification benefit would be to distribute it proportionally to the expected residual risk of each of the subsidiaries, i.e.

$$
\mathrm{DB}_{j}=\mathrm{DB} \cdot \frac{\mathbb{E}\left[\left(X_{j}-F_{X_{j}}^{-1}\left(F_{X_{1}^{c}+\ldots+X_{n}^{c}}\left(u^{*}\right)\right)\right)_{+}\right]}{\mathbb{E}\left[\left(X_{1}^{c}+\ldots+X_{n}^{c}-u^{*}\right)_{+}\right]}, \quad j=1, \ldots, n
$$

\subsection{The absolute problem of economic capital allocation}

In order to illustrate the absolute problem of economic capital allocation, we consider the situation in which the aggregated future losses of the conglomerate is given by $Y$ and analogously $Y_{j}$ for the subsidiaries, and the premium income amounts to $P$ and $P_{j}$ respectively. We naturally assume that $Y=\sum_{j=1}^{n} Y_{j}$ and $P=\sum_{j=1}^{n} P_{j}$ and denote the difference between the future losses and the corresponding premium income by $X$ and $X_{j}$ respectively. Furthermore, we denote by $(i-r)$ the economic cost of raising capital. We consider a risk measure that preserves stochastic dominance, i.e.

$$
X \leq{ }_{1} Y \Rightarrow \rho(X) \leq \rho(Y)
$$

We take as possible examples

$$
\rho_{1}(X, u)=\mathbb{E}\left[(X-u)_{+}\right]+(i-r) u
$$

and (see Kaas et al. (2001))

$$
\rho_{2}(X, u)=\frac{u}{|\log (\varepsilon)|} \log \left(\mathbb{E}\left[\exp \left(\frac{|\log (\varepsilon)|}{u} X\right)\right]\right)+(i-r) u, \quad \varepsilon \in(0,1]
$$

for both of which one can easily prove that if $X=X_{1}+X_{2}$ and $u=u_{1}+u_{2}$ then

$$
\rho(X, u) \leq \rho\left(X_{1}, u_{1}\right)+\rho\left(X_{2}, u_{2}\right)
$$


which expresses the diversification effect on the residual risk level. In order to further simplify expression (15) we consider the economic capitals to be relatively large. Then

$$
\rho_{2}(X, u) \approx \rho_{3}(X, u)=\mathbb{E}[X]+\frac{|\log (\varepsilon)|}{2 u} \operatorname{Var}[X]+(i-r) u .
$$

using only two terms of the Taylor expansion. Suppose that $\mathbb{E}[X]+\mathbb{E}[Y] \leq \frac{2 u}{|\log (\varepsilon)|}$. Then one can easily verify that $\rho_{3}(X, u) \leq \rho_{3}(Y, u)$ in case $X \leq_{1} Y$. However, note that in contrast to $\rho_{2}(X, u)$ the approximation $\rho_{3}(X, u)$ does no longer satisfy the subadditivity property.

To solve the absolute economic capital allocation problem we minimize the risk measure $\rho(X, u)$ with respect to $u$. For the choice of $\rho_{1}(X, u)=\mathbb{E}\left[(X-u)_{+}\right]+(i-r) u$ we obtain the following solutions:

$$
\begin{gathered}
u^{*}=F_{X}^{-1}(1-(i-r)), \\
u_{j}^{* s}=F_{X_{j}}^{-1}(1-(i-r)), \quad j=1, \ldots, n
\end{gathered}
$$

Hence, the optimal amount of economic capital can be calculated by the VaR measure of which the percentile level depends on the economic cost of capital. Now we compare (19) with (8). We find that in order to enforce that the solution to the absolute problem of economic capital allocation for the subsidiaries corresponds to the preference value of the conglomerate, which is the solution to the relative allocation problem, the conglomerate can simply charge a shadow cost of capital $\lambda$ to its subsidiaries. Then, the solution to the absolute allocation problem for subsidiary $j$ is given

$$
u_{j}^{* s}(\lambda)=F_{X_{j}}^{-1}(1-(i-r)-\lambda), \quad j=1, \ldots, n
$$

where $\lambda$ is to be determined as

$$
F_{X}^{-1}(1-(i-r))=\sum_{j=1}^{n} F_{X_{j}}^{-1}(1-(i-r)-\lambda)
$$

or equivalently

$$
\lambda=1-(i-r)-F_{X_{1}^{c}+\ldots+X_{n}^{c}}\left(F_{X_{1}+\ldots+X_{n}}^{-1}(1-(i-r))\right)
$$

Hence, indeed the absolute solution $u_{j}^{* s}(\lambda)$, corresponding to a cost of economic capital $(i-r)+\lambda$, equals the relative allocation $u_{j}^{*}$. It is not difficult to see that typically (not always, see Kaas and Tang (2003) for some interesting counterexamples) $\lambda \geq 0$ and hence $u_{j}^{* s}(\lambda) \leq u_{j}^{* s}$. Thus, both the conglomerate and the subsidiaries incorporated in the conglomerate can use the VaR measure to determine their economic capital, which is highly attractive from a corporate governance point of view. We remark that VaR allocation is consistent with the capital adequacy requirements established by the Basle Capital Accord (see Basle Committee (1988, 1996, 2003)), under which banks currently operate. Subadditivity of the allocation does not play a role in this capital allocation approach. The fact that the order of the percentile is not the same in both (18) and (20) is rather natural. How can one compare a percentile level for the conglomerate with a percentile level for the subsidiaries? 
Next, for the risk measure $\rho_{3}(X, u)=\mathbb{E}[X]+\frac{|\log (\varepsilon)|}{2 u} \operatorname{Var}[X]+(i-r) u$ we obtain

$$
\begin{gathered}
u^{*}=\sqrt{\frac{|\log (\varepsilon)|}{2(i-r)} \operatorname{Var}[X]}, \\
u_{j}^{* s}=\sqrt{\frac{|\log (\varepsilon)|}{2(i-r)} \operatorname{Var}\left[X_{j}\right]}, \quad j=1, \ldots, n \\
u_{j}^{* s}(\lambda)=\sqrt{\frac{|\log (\varepsilon)|}{2(i-r+\lambda)} \operatorname{Var}\left[X_{j}\right]}, \quad j=1, \ldots, n
\end{gathered}
$$

In this situation, because

$$
\sqrt{\frac{|\log (\varepsilon)|}{2(i-r)} \operatorname{Var}[X]} \leq \sum_{j=1}^{n} \sqrt{\frac{|\log (\varepsilon)|}{2(i-r)} \operatorname{Var}\left[X_{j}\right]}
$$

it holds true that $\lambda \geq 0$ if it is determined such that $\sum_{j=1}^{n} u_{j}^{* s}(\lambda)=u^{*}$, and hence a shadow cost is due.

Another interpretation is possible by transforming $\varepsilon$ into $\varepsilon^{\prime}$, representing a larger probability of ruin:

$$
\frac{|\log (\varepsilon)|}{2(i-r+\lambda)}=\frac{\left|\log \left(\varepsilon^{\prime}\right)\right|}{2(i-r)} \Rightarrow \varepsilon \leq \varepsilon^{\prime}
$$

This finding is also natural since within a conglomerate one can intuitively accept a probability of ruin at the subsidiary level that is higher than the probability of ruin at the conglomerate level, which brings out the diversification benefit in another way.

The values of $u_{j}^{* s}(\lambda)$ can be obtained by solving the following system of equations numerically:

$$
\frac{u_{j}^{* s}(\lambda)}{u^{*}}=\frac{\sqrt{\operatorname{Var}\left[X_{j}\right]}}{\sum_{j=1}^{n} \sqrt{\operatorname{Var}\left[X_{j}\right]}}, \quad j=1, \ldots, n
$$

\section{The emergence of virtual economic capital}

Since subsidiaries are juridical entities, one should indeed not only solve the absolute problem of economic capital allocation for the conglomerate, but also for the subsidiaries, when considered as stand alone entities. The latter solutions should then be compared with the solutions to the relative allocation problem of the conglomerate. Using $\rho_{1}(X, u)$ defined in (14) as the risk measure in the absolute allocation problem, we derived in section 2.2 that

$$
u_{j}^{* s}=F_{X_{j}}^{-1}(1-(i-r))
$$

which is to be compared with

$$
u_{j}^{*}=F_{X_{j}}^{-1}\left(F_{X_{1}^{c}+\ldots+X_{n}^{c}}(u)\right)=F_{X_{j}}^{-1}\left(F_{X_{1}^{c}+\ldots+X_{n}^{c}}\left(F_{X_{1}+\ldots+X_{n}}^{-1}(1-(i-r))\right)\right)
$$


using the expectation in the relative allocation problem (7). Clearly, instead of $u_{j}^{*}$ we can also write $u_{j}^{* s}(\lambda)$ as defined in (20), corresponding to a capital cost ( $i-$ $r)+\lambda$. We have seen in section 2.2 that in general (not always) $u_{j}^{*}(\lambda) \leq u_{j}^{* s}$ and hence $\lambda \geq 0$. In case $\lambda<0$, the conglomerate will typically regard its existence as undesirable. Obviously, a subsidiary is willing to pay a positive shadow cost $\lambda$ only if it is compensated by the conglomerate in some sense. Hence, we should consider the diversification benefit for the subsidiaries of being incorporated in the conglomerate.

The cost of risk-bearing of subsidiary $j$ when considered as a stand alone entity is given by

$$
c_{j}^{* s}=(i-r) u_{j}^{* s}+\mathbb{E}\left[\left(X_{j}-u_{j}^{* s}\right)_{+}\right], \quad j=1, \ldots, n
$$

Furthermore, the cost of risk-bearing attributed to subsidiary $j$ when incorporated in the conglomerate construction, but without taking into account the diversification benefit is given by

$$
c_{j}^{* s}(\lambda)=(i-r+\lambda) u_{j}^{* s}(\lambda)+\mathbb{E}\left[\left(X_{j}-u_{j}^{* s}(\lambda)\right)_{+}\right], \quad j=1, \ldots, n
$$

The conglomerate situation becomes more favorable in case the diversification benefits are taken into account. We have seen in (11) that the diversification benefit of the conglomerate construction is given by

$$
\begin{aligned}
\mathrm{DB}= & \sum_{j=1}^{n} \mathbb{E}\left[\left(X_{j}-u_{j}^{* s}(\lambda)\right)_{+}\right]-\mathbb{E}\left[(X-u)_{+}\right]= \\
& (1-\alpha) \sum_{j=1}^{n} \mathbb{E}\left[\left(X_{j}-u_{j}^{* s}(\lambda)\right)_{+}\right], \quad \text { for some } \alpha \in[0,1]
\end{aligned}
$$

Taking into account the diversification benefits, according to a proportional distribution among the subsidiaries, the cost of risk-bearing attributed to subsidiary $j$ is given by

$$
\begin{aligned}
c_{j}^{* D B}(\lambda)= & (i-r+\lambda) u_{j}^{* s}(\lambda)+\alpha \mathbb{E}\left[\left(X_{j}-u_{j}^{* s}(\lambda)\right)_{+}\right]= \\
& (i-r+\lambda) u_{j}^{* s}(\lambda)+\mathbb{E}\left[\left(X_{j}-u_{j}^{* D B}(\lambda)\right)_{+}\right], \quad j=1, \ldots, n
\end{aligned}
$$

Clearly, $c_{j}^{* D B}(\lambda) \leq c_{j}^{* s}(\lambda)$ and $u_{j}^{* D B}(\lambda) \geq u_{j}^{* s}(\lambda)$. The difference between $u_{j}^{* D B}(\lambda)$ and $u_{j}^{* s}(\lambda)$ is what we call the virtual economic capital of subsidiary $j$, i.e.

$$
u_{j}^{* v}(\lambda)=u_{j}^{* D B}(\lambda)-u_{j}^{* s}(\lambda) \geq 0, \quad j=1, \ldots, n
$$

The existence of the diversification benefit and hence of a virtual economic capital should not be ignored by the regulatory authority when assessing the solvency position of each subsidiary.

To determine whether or not the conglomerate construction is beneficial when compared with the stand alone situation, one should consider the difference

$$
\begin{array}{r}
c_{j}^{* D B}(\lambda)-c_{j}^{* s}= \\
(i-r+\lambda) u_{j}^{* s}(\lambda)+\mathbb{E}\left[\left(X_{j}-u_{j}^{* D B}(\lambda)\right)_{+}\right]-(i-r) u_{j}^{* s}-\mathbb{E}\left[\left(X_{j}-u_{j}^{* s}\right)_{+}\right]
\end{array}
$$

which for subsidiary $j$ may or may not be non-positive, depending on the relative dependence structure within the conglomerate and the cost of economic capital. 


\subsection{Generalization with a comonotonic additive risk measure}

We will now generalize the previous results by replacing $\rho_{1}(X, u)$ by a comonotonic additive risk measure. In particular, we specify

$$
\begin{aligned}
\rho_{4}(X, u)=\pi\left((X-u)_{+}\right)+(i-r) u= & \int_{0}^{1} F_{(X-u)_{+}}^{-1}(y) d(1-g(1-y))+(i-r) u= \\
& \int_{u}^{\infty} g\left(1-F_{X}(x)\right) d x+(i-r) u
\end{aligned}
$$

for some strictly increasing and continuous function $g:[0,1] \rightarrow[0,1]$, satisfying $g(0)=0$ and $g(1)=1$. The function $g(\cdot)$ is known as a distortion or probability weighting function. We refer to Yaari (1987) and Wang, Young and Panjer (1997) for an axiomatic characterization of distortion risk measures. It is not difficult to verify that if $u=u_{1}+\ldots+u_{n}$ then

$$
\rho_{4}\left(X_{1}^{c}+\ldots+X_{n}^{c}, u\right)=\rho_{4}\left(X_{1}, u_{1}\right)+\ldots+\rho_{4}\left(X_{n}, u_{n}\right)
$$

and hence $\rho_{4}(X, u)$ is a comonotonic additive risk measure. Minimizing $\rho_{4}(X, u)$ with respect to $u$ yields the following solutions:

$$
\begin{gathered}
u^{*}=F_{X}^{-1}\left(1-g^{-1}(i-r)\right) \\
u_{j}^{* s}=F_{X_{j}}^{-1}\left(1-g^{-1}(i-r)\right), \quad j=1, \ldots, n \\
u_{j}^{* s}(\lambda)=F_{X_{j}}^{-1}\left(1-g^{-1}(i-r+\lambda)\right), \quad j=1, \ldots, n
\end{gathered}
$$

where $\lambda$ is determined such that

$$
\sum_{j=1}^{n} F_{X_{j}}^{-1}\left(1-g^{-1}(i-r+\lambda)\right)=F_{X}^{-1}\left(1-g^{-1}(i-r)\right)
$$

As before, $\lambda$ typically satisfies $\lambda \geq 0$. We remark that $u_{j}^{* s}(\lambda)$ as defined in (39) is not in general equal to the relative allocation $u_{j}^{*}$ obtained by solving

$$
\min _{u_{1}, \ldots, u_{n} \mid \sum_{j=1}^{n} u_{j}=u^{*}} \pi\left(\sum_{j=1}^{n}\left(X_{j}-u_{j}\right)_{+}\right)
$$

Clearly, one may introduce a vector $\left(\lambda_{1}, \ldots, \lambda_{n}\right)$ to establish the equality $u_{j}^{* s}\left(\lambda_{j}\right)=$ $u_{j}^{*}, j=1, \ldots, n$. However, for reasons of simplicity we restrict to a single shadow cost $\lambda$. The diversification benefit of incorporating subsidiaries in a conglomerate construction is then given by

$$
\begin{aligned}
\mathrm{DB}= & \sum_{j=1}^{n} \pi\left(\left(X_{j}-u_{j}^{* s}(\lambda)\right)_{+}\right)-\pi\left(\left(X_{1}+\ldots+X_{n}-u^{*}\right)_{+}\right)= \\
& \pi\left(\left(X_{1}^{c}+\ldots+X_{n}^{c}-u^{*}\right)_{+}\right)-\pi\left(\left(X_{1}+\ldots+X_{n}-u^{*}\right)_{+}\right)
\end{aligned}
$$

It can be verified that for a concave distortion function $g(\cdot)$, the diversification benefit is non-negative in general. Clearly, if a negative diversification benefit occurs, the 
subsidiaries will typically not be willing to pay a positive shadow cost $\lambda$ and a conglomerate construction will appear to be undesirable. In case of a non-negative diversification benefit, we have that

$$
\mathrm{DB}=(1-\alpha) \pi\left(\left(X_{1}^{c}+\ldots+X_{n}^{c}-u^{*}\right)_{+}\right), \quad \text { for some } \alpha \in[0,1]
$$

which may be distributed among the subsidiaries. Hence, the cost of risk-bearing attributed to subsidiary $j$, taking into account the shadow cost $\lambda$ and the diversification benefit according to a proportional distribution, is given by

$$
\begin{aligned}
c_{j}^{* D B}(\lambda)= & (i-r+\lambda) u_{j}^{* s}(\lambda)+\alpha \pi\left(\left(X_{j}-u_{j}^{* s}(\lambda)\right)_{+}\right)= \\
& (i-r+\lambda) u_{j}^{* s}(\lambda)+\pi\left(\left(X_{j}-u_{j}^{* D B}(\lambda)\right)_{+}\right), \quad j=1, \ldots, n
\end{aligned}
$$

Again a virtual economic capital $u_{j}^{* v}(\lambda)=u_{j}^{* D B}(\lambda)-u_{j}^{* s}(\lambda)$ emerges.

\section{The role of economic capital in optimal portfolio se- lection}

In practice, people working in the framework of optimal portfolio selection generally agree that in order to benefit from supplementary returns some surplus capital (call it economic capital) is desirable. Optimal portfolio selection is often performed on the basis of the well-known Markovitz portfolio selection approach (Markovitz (1952, 1959)). An alternative procedure, employing a shortfall constraint has been developed in Basak and Shapiro (2001). In the present investigation, we present an approach that explicitly takes into account the amount of economic capital available and the economic cost of raising it.

We denote by $V$ the future random gain obtained by an investment $\theta_{0}$ in riskfree assets generating a non-random return $r$, and investments $\theta_{j}$ in risky assets $j, j=1, \ldots, m$ with random return $X_{j}$, i.e.

$$
V=\theta_{0} r+\theta_{1} X_{1}+\ldots+\theta_{m} X_{m}
$$

Furthermore, we let $\theta=\theta_{0}+\ldots+\theta_{m}$ denote the total amount invested. The Markovitz portfolio theory considers a utility function

$$
U=V-\iota(V-\mathbb{E}[V])^{2}
$$

where $\iota$ denotes a tolerance level, i.e. a degree of risk aversion. The optimal investment portfolio is then obtained by maximizing

$$
\mathbb{E}[U]=\mathbb{E}[V]-\iota \mathbb{E}\left[(V-\mathbb{E}[V])^{2}\right]
$$

Since the dimension of $\mathbb{E}[V]$ is the monetary unit and the dimension of $\mathbb{E}[(V-$ $\left.\mathbb{E}[V])^{2}\right]$ is the "squared monetary unit", it is reasonable to think of $\iota$ as a tolerance level expressed in " 1 over monetary units". This natural interpretation is often 
overlooked, typically leading to unrealistic results (see e.g. Gerrard, Haberman and Vigna (2003) and Schnieper (2003)). One could choose e.g. $\iota=\alpha / u$ where $\alpha$ is a dimensionless constant and $u$ denotes the amount of economic capital available.

An alternative approach following Markovitz general concept could be to consider the expected gain $\mathbb{E}[\max (V-\theta r, 0)]=\mathbb{E}\left[(V-\theta r)_{+}\right]$on the risky portfolio and deduct from it the cost of the downside risk $\beta \mathbb{E}[\max (\theta r-V, 0)]=\beta \mathbb{E}\left[(\theta r-V)_{+}\right]$, in which $\beta>1$ due to the safety margin which is required in the actuarial framework. Hence, we introduce the following utility function:

$$
U=(V-\theta r)_{+}-\beta(\theta r-V)_{+}, \quad \beta>1
$$

Then,

$$
\mathbb{E}[U]=\mathbb{E}\left[(V-\theta r)_{+}\right]-\beta \mathbb{E}\left[(\theta r-V)_{+}\right], \quad \beta>1
$$

represents the expected utility of the investment strategy $\left(\theta_{0}, \theta_{1}, \ldots, \theta_{m}\right)$ which should be maximized. However, the available economic capital does not play a role in this approach, which is against common sense. To capture the effect of economic capital in the selection procedure, we employ a specific pricing principle (or risk measure) for the downside risk, in particular we use a pricing principle expressed in terms of the economic capital, namely

$$
\frac{u}{|\log (\varepsilon)|} \log \left(\mathbb{E}\left[\exp \left(\frac{|\log (\varepsilon)|}{u}(\theta r-V)_{+}\right)\right]\right), \quad \varepsilon \in(0,1]
$$

based on a ruin criterion (see e.g. Kaas et al. (2001)). Introducing this pricing principle into the expected utility expression yields

$$
\mathbb{E}[U]=\mathbb{E}\left[(V-\theta r)_{+}\right]-\frac{u}{|\log (\varepsilon)|} \log \left(\mathbb{E}\left[\exp \left(\frac{|\log (\varepsilon)|}{u}(\theta r-V)_{+}\right)\right]\right)
$$

Suppose that $u$ is relatively large. Then the above expression can be approximated by

$$
\begin{array}{r}
\mathbb{E}\left[(V-\theta r)_{+}\right]-\mathbb{E}\left[(\theta r-V)_{+}\right]-\frac{|\log (\varepsilon)|}{2 u} \operatorname{Var}\left[(\theta r-V)_{+}\right]= \\
\mathbb{E}[V]-\theta r-\frac{|\log (\varepsilon)|}{2 u} \operatorname{Var}\left[(\theta r-V)_{+}\right]
\end{array}
$$

using only the first two terms of the Taylor expansion. When maximizing the expected utility of the investment strategy, we naturally restrict to solutions $\left(\theta_{0}^{*}, \theta_{1}^{*}, \ldots, \theta_{m}^{*}\right)$ for which the value of the objective function (51) is non-negative. It follows that the economic capital $u$ should be large enough in order to construct an optimal portfolio. For the case of only 1 risky asset, the optimal portfolio with total amount invested equal to $\theta=\theta_{0}+\theta_{1}$ is given by

$$
\theta_{1}^{*}=\theta-\theta_{0}^{*}=\frac{u}{|\log (\varepsilon)|} \cdot \frac{\mathbb{E}\left[X_{1}\right]-r}{\operatorname{Var}\left[\left(r-X_{1}\right)_{+}\right]}
$$

Observe that the amount invested in the risky asset is naturally increasing in the amount of economic capital. We remark that the expected utility in expression (51) 
does not take into account the economic cost of capital $(i-r) u$. Clearly, for a given level of economic capital the solution $\left(\theta_{0}^{*}, \theta_{1}^{*}, \ldots, \theta_{m}^{*}\right)$ is independent of whether or not the economic cost of capital is taken into account. Indeed, adding a constant $-(i-r) u$ to the objective function (51) does not affect the solution. However, let us now consider the case of a variable level of economic capital. In that case the expected utility maximization problem is given by

$$
\max _{\left(\theta_{0}, \theta_{1}, \ldots, \theta_{m}\right), u \mid \sum_{j=0}^{m} \theta_{j}=\theta} \mathbb{E}[V]-\theta r-\frac{|\log (\varepsilon)|}{2 u} \operatorname{Var}\left[(\theta r-V)_{+}\right]-(i-r) u
$$

For the case of only 1 risky asset and $\theta=\theta_{0}+\theta_{1}$, we obtain the following optimal investment strategy:

$$
\theta_{1}^{*}=\theta-\theta_{0}^{*}= \begin{cases}\theta, & \mathbb{E}\left[X_{1}\right]-r-\sqrt{2|\log (\varepsilon)|(i-r) \operatorname{Var}\left[\left(r-X_{1}\right)_{+}\right]}>0 \\ \alpha \theta, \alpha \in[0,1], & \mathbb{E}\left[X_{1}\right]-r-\sqrt{2|\log (\varepsilon)|(i-r) \operatorname{Var}\left[\left(r-X_{1}\right)_{+}\right]}=0 \\ 0, & \mathbb{E}\left[X_{1}\right]-r-\sqrt{2|\log (\varepsilon)|(i-r) \operatorname{Var}\left[\left(r-X_{1}\right)_{+}\right]}<0\end{cases}
$$

not allowing short-selling and hence restricting to $\theta_{j} \geq 0, j=1,2$. Furthermore, the optimal amount of surplus capital is given by

$$
u^{*}=\theta_{1}^{*} \sqrt{\frac{|\log (\varepsilon)|}{2(i-r)} \operatorname{Var}\left[\left(r-X_{1}\right)_{+}\right]}
$$

Hence, we find that in case of only one risky asset, the optimal investment strategy is a boundary solution. Indeed, if the risk premium $\mathbb{E}\left[X_{1}\right]-r$ is sufficiently large, the total amount $\theta$ should be invested in the risky asset. Otherwise, it is optimal to invest only in the risk-free asset and to hold no surplus capital. Whether or not the risk premium is sufficiently large, naturally depends on the economic cost of capital.

\section{Conclusions}

We proposed a solution to both the absolute and the relative problem of economic capital allocation based on a residual risk consideration. The solutions take into account the economic cost of capital. We compared the relative allocation among subsidiaries with the absolute allocation for subsidiaries when considered as stand alone entities. We demonstrated the existence of a virtual economic capital for subsidiaries in a conglomerate, which should be recognized as such by a regulatory authority. The positive value difference between the solvency margin as imposed for rating considerations (e.g. a 99.975\% level) and the optimal amount of economic capital as obtained in this paper, may be used to generate supplementary returns. Hence, we considered the role of economic capital in optimal portfolio selection. We found that for a variable level of economic capital and the case of only one risky asset a boundary solution is obtained: it is optimal to invest either in the risky asset or in the risk-free asset. 


\section{References}

[1] Basak, S. and A. Shapiro (2001). "Value-at-risk-based risk management: optimal policies and asset prices," Review of Financial Studies, 14, 371-405.

[2] Basle Committee on Banking Supervision (1988). "International convergence of capital measurement and capital standards."

[3] Basle Committee on Banking Supervision (1996). "Amendment to the capital accord to incorporate market risks."

[4] Basle Committee on Banking Supervision (2003). "The new Basel capital accord."

[5] Bühlmann, H. (1970). Mathematical Methods in Risk Theory, Berlin: Springer Verlag.

[6] Casualty Actuarial Society (1999). "Principles underlying actuarial science."

[7] Dhaene, J., M. Denuit, M.J. Goovaerts, R. Kaas and D. Vyncke (2002a). "The concept of comonotonicity in actuarial science and finance: theory," Insurance: Mathematics and Economics, 31, 3-33.

[8] Dhaene, J., M. Denuit, M.J. Goovaerts, R. Kaas and D. Vyncke (2002b). "The concept of comonotonicity in actuarial science and finance: applications," Insurance: Mathematics and Economics, 31, 133-161.

[9] Gerrard, R., Haberman, S. and E. Vigna (2003). "Optimal investment choices post retirement in a defined contribution pension scheme," Proceedings of the 13th AFIR Conference Maastricht.

[10] Gerber, H.U. (1979). An Introduction to Mathematical Risk Theory, Huebner Foundation Monograph 8, distributed by R.D. Irwin, Illinois: Homewood.

[11] Goovaerts, M.J., F. de Vylder and J. Haezendonck (1984). Insurance Premiums, Amsterdam: North Holland Publishing.

[12] Goovaerts, M.J., J. Dhaene and R. Kaas (2003). "Capital allocation derived from risk measures," North American Actuarial Journal, 7, 44-59.

[13] Kaas, R., M.J. Goovaerts, J. Dhaene, and M. Denuit (2001). Modern actuarial risk theory, Dordrecht: Kluwer Academic Publishers.

[14] Kaas, R. and Q. Tang (2003). "Some useful counterexamples regarding comonotonicity," submitted.

[15] Markovitz, H.M. (1952). "Portfolio selection," Journal of Finance, 7, 77-91.

[16] Markovitz, H.M. (1959). Portfolio Selection: Efficient Diversification of Investments, New York: John Wiley. 
[17] Schnieper, R. (2003). "Capital and asset allocation," Proceedings of the 34th ASTIN Conference Berlin.

[18] Wang, S.S., V.R. Young and H.H. Panjer (1997): "Axiomatic characterization of insurance prices," Insurance: Mathematics and Economics, 21, 173-183.

[19] Yaari, M.E. (1987): "The dual theory of choice under risk," Econometrica, $55,95-115$. 